\section{Géneros}

Multidisciplinary Journal of Gender: Studies

\section{Hipatia Press \\ www.hipatiapress.com}

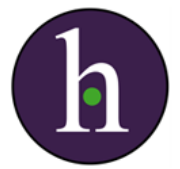

Instructions for authors, subscriptions and further details:

http://generos.hipatiapress.com

\title{
Género, Salud y Autocuidado en Académicas Universitarias
}

María del Rocío Guzmán Benavente ${ }^{1}$

Graciela Polanco Hernández ${ }^{2}$

Karla María Reynoso Varga ${ }^{1}$

Cosme Francisco Maldonado Rivera ${ }^{1}$

1) Universidad Juárez del Estado de Durango, México

2) Universidad Iberoamericana, Ciudad de México, México

Date of publication: June $25^{\text {th }}, 2018$

Edition period: June - October 2018

To cite this article: Guzmán, M.R.; Polanco, G.; Reynoso, K.M.;

Maldonado, C.F. (2018). Género, Salud y Autocuidado en Académicas

Universitarias. Multidisciplinary Journal of Gender Studies, 7(2), 1657-1680.

doi: 10.17583/generos.2018.3089

To link this article: http://dx.doi.org/10.17583/generos.2018.3089

\section{PLEASE SCROLL DOWN FOR ARTICLE}

The terms and conditions of use are related to the Open Journal System and to Creative Commons Attribution License (CC-BY). 
GÉNEROS -Multidisciplinary Journal of Gender Studies Vol. 7 No.2 June 2018 pp. 1657-1680

\section{Gender, Health and Self-care in University Women Professors}

María del Rocío Guzmán

Karla María Reynoso

Cosme Francisco Maldonado

Universidad Juárez del Estado de Durando
Graciela Polanco

Universidad Iberoamericana

\section{Abstract}

The study aimed to understand the women professor's health care experience, considering personal, family and occupational aspects. It was a qualitative, phenomenological, and biographical study. 15 teachers from a Mexican University were interviewed. The information was structured in three categories: 1) Multitasking stress; 2) Diseases and medical conditions; and 3) Psychological supports. It was found that teachers developed psychological mechanisms (depersonalization, pain normalization, and rationalizations), which allow coping with the multitasking stress, but resulting in self-care neglect and disease. The stories showed health vulnerability and how individual psychosocial supplied tools to cope with health problems. A gender perspective is discussed as a necessity in the woman professor's health assessment. It is questioned the institution role, and it is required a comprehensive approach about inequalities in health and academic productivity.

Keywords: women professors, health, illness, self-care, gender. 


\section{Género, Salud y Autocuidado en Académicas Universitarias}

María del Rocío Guzmán

Karla María Reynoso

Cosme Francisco Maldonado

Universidad Juárez del Estado de Durando
Graciela Polanco

Universidad Iberoamericana

\section{Resumen}

El estudio se enfocó en comprender la vivencia del cuidado de la salud en mujeres académicas, contemplando aspectos personales, familiares y laborales. Fue cualitativo, fenomenológico y biográfico. Se entrevistaron a 15 académicas de una universidad mexicana. La información se revisó a partir de tres categorías de análisis: 1) Estrés multitareas; 2) Enfermedades y dolencias; y 3) Soportes psicosociales. Se encontró que las profesoras desarrollaron mecanismos psicológicos (despersonalización, normalización del dolor, y racionalizaciones) para lidiar con el estrés multitareas, pero incurrieron en negligencia en su autocuidado y padecimiento de enfermedades. Los relatos expusieron vulnerabilidad sanitaria y cómo los soportes psicosociales de tipo personal proveyeron las herramientas para enfrentar los problemas de salud. Se discute necesaria una lente de género en la valoración de la salud de las académicas. Se cuestiona el papel de las instituciones, y se precisa un enfoque integral sobre las desigualdades de salud y desarrollo académico.

Palabras clave: académicas, salud mental y física, enfermedades, autocuidado, género. 



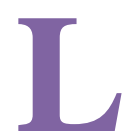

a construcción histórica - y sociocultural- del género como elemento constitutivo de las relaciones entre mujeres y hombres y sus implicaciones de poder (Scott, 2013), permea la micro y la macroesfera de la sociedad a través del mercado de trabajo, del sistema educativo, de los medios de comunicación, del aparato político, de la familia, de las relaciones interpersonales, e incide en la salud de unas y otros (Alderete, \& Franco, 2006).

La salud es un derecho fundamental de todo ser humano sin distinción de raza, religión, ideología política, condición económica o social. Sin embargo, existen diferencias entre las mujeres y los hombres en cuanto a los factores que la determinan y su carga de morbilidad con repercusiones en la esfera sanitaria (OMS, 2002).

La salud del personal académico (mujeres y hombres) en las universidades se ve afectada por diversas condiciones laborales, como ocurre con otros trabajadores del sector terciario (Arquero, \& Donoso, 2013; Magaña, \& Sánchez, 2008). El estrés crónico frecuentemente desemboca en "agotamiento profesional" o "síndrome de burnout". Cuadro caracterizado por desgaste emocional, despersonalización y falta de logro (Maslach, 2003), con consecuencias físicas negativas, como fatiga crónica, dolor de cabeza, insomnio, hipertensión; cognitivo-emocionales expresadas en sentimientos de infelicidad, ansiedad, desatención, dificultad para tomar decisiones; organizacionales como el ausentismo, falta de continuidad en las actividades, deterioro en la calidad del trabajo; y familiares manifiestas en relaciones deterioradas (Moreno, González, \& Garrosa, 2002).

Aún son insuficientes las investigaciones acerca de los costos personales que genera ese tipo de problemas en el personal académico para conocer los efectos del estrés laboral, el agotamiento, la frustración, la falta de satisfacción laboral, y muchos otros aspectos que repercuten en el desempeño de las actividades de docencia e investigación (Barraza y Romero, 2011).

Ser mujer es un determinante psicosocial de desigualdad e inequidad que introduce factores agravantes en la salud (Esteban, 2003; Palomino, Grande, \& Linares, 2014). En la universidad, las académicas viven con tensiones que ajustan distinto a las de sus pares varones. Además del estrés por las presiones laborales, también experimentan fuertes conflictos en la conciliación del trabajo con la familia (Tena, Muñoz, \& López, 2012; Tena, 
Rodríguez, \& Jiménez, 2010) ante las responsabilidades del hogar, de la pareja, de la crianza de las/los hijos y del cuidado de otros familiares dependientes, al mismo tiempo que atienden la carrera laboral (Guzmán, Polanco, Rocha, \&Turnbull, 2016).

Esos esfuerzos afectan el bienestar de las mujeres, tal como lo indican investigaciones de corte cuantitativo y cualitativo enfocadas a estudiar los efectos de las sobrecargas por el desempeño de sus múltiples roles. Sobre esa línea, Blanco y Feldman (2000) encontraron que, académicas con mayores cargas en la planificación de las actividades doméstico-familiares sufrieron más ansiedad y depresión y, consecuentemente, presentaron peor salud mental, sobre todo cuando sintieron menor apoyo de sus parejas.

Otros estudios señalan que el efecto multi rol puede ser favorable o no para la salud de las académicas dependiendo de la calidad del desempeño de los roles tanto en el ámbito laboral como en el doméstico-familiar. Por ejemplo, el trabajo gratificante actúa positivamente en la autoestima de las mujeres cuando se sienten apoyadas laboralmente. Esta condición les genera menos ansiedad en sus roles como madres, esposas y trabajadoras. Frente a mayores demandas laborales, sufren mayores niveles de estrés y depresión, sobre todo cuanto se perciben negativamente en su rol de madres; ya que la percepción de ser una buena madre juega un papel destacado en la valoración que las mujeres hacen de sí mismas (Gómez, 2004; Gómez, Pérez, Feldman, Bajes, \& Vivas, 2000).

Gómez, Perilla y Hermosa (2015) también encontraron que, mujeres académicas sufren más tensiones laborales e incluso pueden presentar trastornos depresivos cuando se viven en conflicto con el trabajo y la familia. En México, la depresión en las mujeres ha llegado a ocupar el primer lugar en enfermedades mentales (González-Pier, et al., 2006) y la tercera causa en la carga de enfermedades padecidas (OMS, 2001).

Los problemas mentales que padecen las mujeres trabajadoras se han relacionado con las condiciones laborales que suelen entrar en conflicto con los compromisos familiares y su derivación en experiencias de agotamiento emocional, así como menor eficacia profesional. Algunos estudios con trabajadoras de diferentes niveles ocupacionales encontraron que los apoyos sociales en el trabajo son fuentes protectoras para la salud y el bienestar, ofreciendo apoyo emocional y práctico y un mayor control en el proceso de 
la toma de decisiones (Feldman, \& Saputi, 2007; Gómez, Pérez, Feldman, Bajes, \& Vivas, 2000).

En Estados Unidos ciertas investigaciones han estudiado la relación del trabajo con la familia en el ámbito de la educación superior para conocer cómo les facilita o no el desarrollo de la carrera académica. Los estudios indican que las mujeres han reportado mayores niveles de conflicto al asumir las principales labores relacionadas con la crianza y el cuidado del hogar. También describen cómo, en gran parte de las universidades, las políticas laborales no favorecen el desarrollo académico de quienes deciden ser madres (Armenti, 2004; Gómez, Perilla, \& Hermosa, 2015; Probert, 2005; Reddick, Rochlen, Grasso, Reilly, \& Spikes, 2012).

Las mujeres trabajadoras continúan con la mayor carga de tareas en el hogar debido a que la participación de los hombres es aún menor (Blanco, \& Feldman, 2000; Wiersma, \&Van Den Berg, 1991). En ese sentido, la doble presencia es un problema estructural que genera vivencias estresantes en las académicas (Guzmán, Polanco, Rocha, \& Turnbull, 2016).

Las diferencias en el uso del tiempo entre mujeres y hombres reflejan desigualdades en la disposición del tiempo libre (personal). De acuerdo con Murillo (2006), el tiempo privado se asume de forma diferente entre unas y otros. Las mujeres han confundido el tiempo personal con el tiempo doméstico, mientras que para ellos representa un tiempo que se dan a sí mismos (en Alcañiz, et al., 2014).

En nuestro país, las mujeres invierten en las actividades domésticas 29.8 horas a las semana en promedio; los hombres, 9.7 horas. Ellas triplican el tiempo registrado por ellos (INEGI, INMUJERES, 2014). Las horas invertidas por unas $\mathrm{y}$ otros indican disparidades en cargas $\mathrm{y}$ responsabilidades, con el riesgo de que las mujeres sufran más problemas de salud por mayor acumulación de estrés, así como desgaste mental y físico (Gómez, et al., 2000). En relación a ello, algunos resultados de encuestas epidemiológicas han encontrado que la depresión, la ansiedad y las quejas somáticas son más frecuentemente en las mujeres (OMS, 2004).

El problema de fondo tiene que ver, por un lado, con un modelo laboral que impone tensiones ocultas por las contradicciones del sistema de acumulación de capital que contraviene el sostenimiento de la vida (Carrasquer, 2009) y, por otro, porque al privatizarse el cuidado de la familia como un asunto de carácter personal y de las mujeres, ni el colectivo 
masculino, ni el estado como administrador de políticas públicas a favor de los grupos sociales han asumido del todo su corresponsabilidad ante un asunto de índole psicosocial, cultural y político-estructural (Guzmán, et al., 2016).

La Organización Panamericana de la Salud (OPS), admite que una persona es saludable cuando logra un desarrollo equilibrado de la vida en sus relaciones interpersonales, familiares y de integración social (en términos comunitarios y económicos) como base para un funcionamiento eficaz. La superación de enfermedades y el desarrollo de estados mentales positivos son factores protectores de la salud psicológica (Taylor, Kemeny, Reed, Bowel, \& Gruenewald, 2000, en Vázquez, et al., 2009).

Desde esa perspectiva, la autoatención (o autocuidado) es un aspecto que comprende actitudes y acciones enfocadas al bienestar. Significa capacidad de explicar, atender, controlar, aliviar, aguantar, curar, solucionar o prevenir procesos que afectan la salud en términos reales o imaginarios. Se relaciona con los hábitos personales, la actividad física y las redes de apoyo social como la propia familia y los amigos, entre otros recursos y prácticas necesarias para la salud de las personas (Berenzon-Gorn, Saavedra-Solano, \& Alanís-Navarro, 2009).

Al reconocer la importancia que adquiere el autocuidado para la salud, ya que ésta es un derecho humano para el bienestar socio-emocional y físico de las personas; y tomando en cuenta que el género es un determinante social de la salud (Palomino, Grande, \& Linares, 2014), el presente estudio se propuso comprender cómo se viven las académicas en el cuidado de su salud, en relación con algunas de sus experiencias de desarrollo personal, familiar y de carrera laboral.

\section{Metodología}

El estudio fue cualitativo; de carácter fenomenológico y biográfico (Bertaux, 1988; Gadamer, 2012), así como de análisis temático. El método fenomenológico permitió abordar la realidad social y humana en busca de un tratamiento más personal e intersubjetivo (relación yo/tú), lo que ha favorecido la emergencia del sentido de vida de las participantes. De esa forma, quien investiga puede llegar a descubrir aquello oculto e integrar la unidad (Barbera \& Inciarte, 2012). 
Desde el método biográfico, las entrevistas en profundidad permitieron el acercamiento con las participantes y la interpretación de sus relatos, reivindicando lo autobiográfico como enfoque metodológico y no solo como herramienta o técnica (Bertaux, 1988), al posibilitar el reconocimiento de significados y contextos de significado de lo individual como parte de lo social, de sus estructuras y de sus normas (Díaz, 1999).

La muestra estuvo integrada por 15 mujeres académicas de tiempo completo de una universidad pública mexicana. Las participantes contaban con un perfil deseable en docencia e investigación y formaban parte del Programa para el Desarrollo Profesional Docente (PRODEP) de la Secretaría de Educación Pública (SEP); cuatro de ellas también pertenecían a Nivel I del Sistema Nacional de Investigadoras (SNI) del Consejo Nacional de Ciencia y Tecnología (CONACyT). Formaron parte de una investigación más amplia acerca de la construcción subjetiva de identidades desde el ámbito personal-familiar y su incidencia en la carrera laboral. Cuando fueron entrevistadas tenían entre 34 y 63 años de edad; 7 de ellas eran casadas; 2, divorciadas; 2 más solteras y 1 viuda. La mayoría tiene entre uno y tres hijos.

Mediante el análisis de relatos se intentó responder a la pregunta relacionada con la salud y el autocuidado de las académicas, tema emergente en la investigación de la que deriva el presente análisis. El tópico de la salud apareció en los encuentros con las primeras académicas entrevistadas, por lo que se decidió integrarlo para profundizar en él, tratando de comprender la vivencia que las participantes han experimentado alrededor de ésta.

Las entrevistas de las que se obtuvieron los relatos duraron aproximadamente dos horas en promedio con cada participante. Se llevaron a cabo en distintos lugares acordados con ellas previamente; algunas, en sus centros de trabajo; otras, en sus casas y otros lugares públicos. La mayoría de las entrevistadas mantuvo abierta la posibilidad de nuevos encuentros u otras formas de comunicación como el correo electrónico y los mensajes telefónicos para integrar la información que hiciera falta. La disposición de las participantes fue siempre de apertura y colaboración.

Para guardar su anonimato, se emplearon nombres ficticios; se editaron y eliminaron datos personales que pudieran contravenir la confidencialidad de su identidad. Se obtuvo su consentimiento para la 
grabación, transcripción, análisis y publicación de los resultados de la investigación.

\section{Resultados y discusión}

El estudio pretendió conocer las vivencias de las académicas desde algunos sucesos acontecidos en su desarrollo personal-familiar y laboral en relación a su salud y autocuidado. Para llevar a cabo el análisis a sus procesos de subjetivación, se construyeron tres categorías: 1) Estrés multitareas; 2) Dolencias y enfermedades, y 3) Soportes psicosociales.

La vida de las participantes ha atravesado por distintas experiencias en las que la salud se tiñe como marca de género (Alderete, \& Franco, 2006) desde la cual las mujeres asumen las responsabilidades de planificación y gestión doméstico-familiar como condición para mantenerse en el mundo del trabajo. Esas tareas las han llevado a experimentar una cotidianidad con ansiedad y estrés e incluso con estados depresivos (Blanco, \& Feldman, 2000; Gómez, 2004; Tena, et al., 2012).

Los malestares psicológicos y físicos se han asociado a la condición de ser mujeres doble-presentes (Guzmán, et al., 2016) para hacer compatibles las demandas del trabajo con las de la vida familiar. Las experiencias de este grupo de académicas han transitado entre dolores de cabeza, dolores musculares, problemas de presión, taquicardias, alopecia, dolores de estómago, parálisis por tensión, pérdida de visión, entre otras. El sobre peso, apareció como un problema que desencadena o agrava otras enfermedades (diabetes, problemas ortopédicos, obesidad, hipertiroidismo, presión reactiva, entre otras). Esos malestares y enfermedades se asemejan a los que han padecido otras académicas por cargas del trabajo, tareas de la casa y la familia (Tena, Muñoz, \& López, 2012) las cuales han detonado en estrés multitareas.

\section{Estrés multitareas}

Los múltiples roles y la calidad con la que son desempeñados por las mujeres trabajadoras han sido fuente de estrés y conflictos que afectan negativamente su salud física y su bienestar psicológico (Phillips, \& Mc Cartney, 1991, en Gómez, et al., 2000; Gómez, 2004; Feldman, \& Sapulti, 
2007; Tena, Rodríguez, \& Jiménez, 2010). Sumados a los roles domésticos y familiares en la vida privada, la academia implica actividades de docencia, gestión, investigación, tutoría académica, dirección de tesis, vinculación y difusión. El avance en esta profesión exige producción individual y colectiva, así como actualización y habilitación del posgrado.

Este último se ha presentado no como una demanda única a resolver, sino también como un periodo colmado de eventos estresantes que implica adaptación, sobrecarga y desgaste para estas académicas. Cuando se cursa en condiciones institucionales poco favorables, puede llegar a producir estados de agotamiento (Gómez, Feldman, Bajes y Vivas, 2000), tal como lo ilustra el siguiente relato.

(...) Los primeros tres semestres (del doctorado) muy padre, pero después, "pooom" (...) ahí fue donde dije, si patino (ahí me quedo) (...), aun así yo cumplía en el sindicato, venía a dar mis clases, asistía a una que otra junta, hacía mi trabajo de evaluación, o sea, yo trataba de cumplir acá y acá (y eso), acabó, acabó con mi salud (...) (Ana, divorciada, un hijo).

La acumulación de estrés coloca a las mujeres en situación de riesgo para la salud física y mental. Los malestares psíquicos se han vinculado íntimamente a malestares físicos. Como se puede deducir del siguiente relato, el proceso psicobiológico del estrés torna vulnerables a las académicas en términos de salud.

(...) me sentía mal, con dolores de cabeza, tenía problemas de presión, de presión arterial, y se presentaron por toda la situación de estrés, y todo lo que esto involucra. Era por presión reactiva, así es como lo calificó el médico, debido al estrés (Elvira, casada, dos hijos).

La vulnerabilidad se acentúa cuando ellas postergan la atención de sus malestares, síntomas de enfermedad o alteraciones en sus estados de ánimo. Los relatos hicieron presente la despersonalización, una de las características del agotamiento profesional (Maslach, 2003). En el caso de las participantes, ésta parece extenderse a sí mismas, expresada en la negligencia hacia su salud y la negación de las propias necesidades 
humanas; y como lo muestra el siguiente relato, no cede ni ante las evidencias ni ante la confrontación.

(...) por supuesto, atiendes a todo lo demás, menos a ti (...). Sí, me dice (mi mamá), ándale hija y ¿por qué tú no? (...) ¿por qué tú no entras con el doctor?, y (yo digo) no; yo después, yo después (...), también está esa parte de dejarlo todo para después (Elvira, casada, dos hijos).

Se genera una espiral ascendente: no se atienden los síntomas de enfermedad porque se está estresado y estar estresado genera síntomas de enfermedad. Hay racionalizaciones que permiten negar el problema, continuar el estilo de vida y el ritmo de trabajo. En contradicción al deterioro de su salud física y/o mental, algunas académicas han asumido ser "saludables".

(...) Afortunadamente he tenido buena salud, creo que hasta la fecha, tengo buena salud, a excepción que me afecta mucho el estrés, cuando hay sobrecarga de trabajo, sí me afecta mucho el estrés (...) (Sol, divorciada, tres hijos).

Sin embargo, la despersonalización y las racionalizaciones no evitan la disrupción orgánica. La desatención tiene consecuencias; con el paso del tiempo, las enfermedades aparecen o se acentúan en momentos de intenso trabajo y desatención personal. En los casos de mayor sobreimplicación laboral, las molestias y enfermedades han cobrado importancia hasta el momento en que las académicas se han visto incapacitadas para seguir laborando. Esperar hasta un periodo crítico ha tenido altos costos, dificultado la evolución clínica con secuelas a largo plazo.

(...) lo que ahorita tengo de enfermedades son de ese tiempo, ¿verdad? y alguna vez me quedé hasta que no pude dar un paso de la tensión que tenía (...). Me quedé paralizada como quince días (...) era cuestión de estrés (...) muchas tensiones (Cristina, viuda, tres hijos, dos nietos).

Otro mecanismo que se ha desprendido de la vivencia estresante es una especie de normalización del dolor. Las participantes han aprendido a 
convivir con molestias físicas y emocionales, de tal suerte que su presencia no se percibe como extraordinaria; se vuelve parte de la vida, genera tolerancia. De esta manera, los síntomas pueden evolucionar hasta formar cuadros severos. El relato siguiente muestra el alto riesgo que corren estas mujeres al normalizar el dolor y no atenderse a tiempo.

El mayor problema que te puedo comentar (fue), una lesión, una fractura en el brazo, (y) no atendérmela, por no saber que era fractura, pese a que no podía dormir (...). No me di cuenta y nunca me atendí esa lesión. Hasta, ya a los meses que era insoportable que voy a ver, y que me dice el terapeuta, el fisioterapeuta, que necesita una radiografía. Me checa y me dice, mire aquí estaba la fractura (...) pegó mal, por eso tiene ese problema en su hombro (Elvira, casada, dos hijos).

Paradójicamente los mismos mecanismos psicológicos que en un inicio resultaron adaptativos y les permitieron avanzar en su carrera, al volverse crónicos han perjudicado su salud.

El estrés se ha vivido como "algo atorado", que irrumpe en el cuerpo, que "cobra facturas". Los síntomas físicos se perciben como un indicador que entorpece el trabajo y el transcurrir de la vida cotidiana. Han podido observar los efectos de su estilo de vida hasta que su propio comportamiento las ha confrontado.

(hay que) sortear las dificultades o lidiar con lo que te digo de la cuestión de las enfermedades (...). Es que vives en un estrés así como muy fuerte, ¿verdad? Yo después de mucho tiempo me di cuenta que todos los días tenía dolor de estómago, no un dolor así como dolor, sino como algo atorado, ¿verdad? Es que corres todo el día (...), un día me fui a trabajar con el vestido puesto al revés (...) y dices, ¡Qué es esto! O sea, no me pude ni siquiera ver al espejo a ver cómo salía. Ese día dije, ¡No puede ser! No puede ser que corras a ese ritmo (...) dejas al niño en la escuela, dejas medio el desayuno, medio la comida, medio la ropa, medio lo que le dices a la muchacha que te ayude y sales corriendo (Cristina, viuda, tres hijos, dos nietos).

Parte del porqué las académicas se han despersonalizado a sí mismas es que su tiempo, al estar más condicionado socioculturalmente que el de los 
hombres, resulta insuficiente para invertirlo en su cuidado - por ejemplo, las mujeres pueden estar sometidas a la pareja o a las/los hijos. Cuando se sacrifica el tiempo personal para sí mismas, se refleja como desigualdades en el trabajo -doméstico y extra doméstico-. Porque el tiempo privado se asume de forma distinta entre unas y otros (Murillo, 2006). Ellos lo emplean para sí mismos; las mujeres suelen confundirlo con el tiempo doméstico-familiar (en Alcañiz, et al., 2014), contrapuesto al del trabajo fuera de casa y a las exigencias de la formación académica. Disponer de tiempo personal solo ha sido posible robándole horas al sueño.

(...) desde el momento mismo que no hiciste las horas de sueño necesarias, porque durante ese tiempo es cuando le dedicas a muchas de las cosas que tienes profesionalmente. Para tener muchos de los logros que tenemos, son en las horas que todos están dormidos (Elvira, casada, dos hijos).

Para algunas, como en el siguiente relato, contar con tiempo para sí mismas solo ha sido factible cuando las obligaciones familiares han disminuido o concluido. Lo que ocurre, generalmente, cuando los hijos han crecido o cuando la pareja se ha ido.

(...) ahora tengo tiempo por ejemplo de hacer comidas como mucho más sanas $(\ldots)$, hacer la dieta que necesito (...) porque tengo diabetes. Hacer el ejercicio que necesito (...). Puedo pagar un tratamiento por fuera (...). En un tiempo, pues no puedes hacer tampoco ejercicio porque a qué hora, o sea a qué hora vas al gimnasio (...). A lo mejor caminas un ratito pero nada más, ¿verdad? Pero así como un programa de ejercicios que necesitas (no se ha podido), en el caso por ejemplo mío, que en la cuestión de la diabetes tiene mucho que ver (...) (Cristina, viuda, tres hijos, dos nietos).

El estrés multitareas y la sobreimplicación ha provocado agotamiento emocional y físico a este grupo de académicas, sobre todo cuando han desatendido su salud por atender los requisitos académicos y las necesidades de otros/as. Los descuidos, con el paso del tiempo, también han derivado en enfermedades con síntomas severos, poniendo al descubierto la vulnerabilidad y el riego en que puede estar la salud de estas mujeres. 


\section{Enfermedades y dolencias}

Uno de los problemas que han padecido estas académicas con mayor frecuencia es el sobrepeso. Con él han caminado a ratos entre malestares y dolencias, soslayando su importancia al priorizar, sobre todo, las necesidades de las y los hijos, y posponiendo su atención o negándose a enfermar porque no hay tiempo para ello.

Me he descuidado, debería hacer ejercicio (...) no me da tiempo (...). No soy enfermiza afortunadamente, igual tengo la teoría de que inconscientemente como (mi hijo menor) me necesita no me enfermo" (Josefina, casada, dos hijos).

(...) Lo que no he logrado es bajar de peso, eso sí es lo único que no he logrado. Este, $\mathrm{mmm}$, pero que haya tenido alguna enfermedad fuerte, o sea, no! De vez en cuando me dan taquicardias (...) Me dijeron que era por estrés y son esporádicas (...), pueden llegar de repente pero, no, hasta ahorita no tengo nada serio. Pero sí me han recomendado que baje de peso (...). Estoy haciendo ejercicio, ahorita tengo más tiempo, (porque) mis hijas no están aquí (...), y pues yo creo que es lo único que a veces me preocupa (...). He tenido buena salud, creo que soy una persona fuerte. Fuerte, mentalmente y creo que soy una persona fuerte físicamente (...) (Hipatía, casada, dos hijas)

Minimizar la importancia del sobrepeso y de otras enfermedades ha llevado a las participantes a desatenderse, ponderando el valor de la académica y la investigación.

(...) Mi único problemilla es que tengo sobrepeso (...). Tengo problemas ortopédicos que digo, son mecánicos. A mí me preocupa más un problema sistémico metabólico que mis rodillas ¡Están deterioradas! (...)" (Carlota, casada, dos hijos y un nieto).

"Gordura, ya no podía caminar de tanto estar sentada (...) daba la clase y me sentaba, me sentaba porque ya no podía estar parada, me subía a mi cubículo a seguirle, ya no caminaba (...). Yo tengo problema de hipertiroidismo y descuidé mi ida al médico, descuidé mi tratamiento, descuidé mi sueño, (...) a veces comía, a veces no (...), cambié mi sistema de alimentación (...) mi carácter cambió, ¿no te 
digo? Mi vista se acabó, ya no veía, mi voz tartamudeaba al hablar, (tenía) dolores en todo mi cuerpo y mi columna. Yo veía que mi estómago se me sentaba ya en mis piernas (...) ¡Horrible, horrible! (...) (Anna, divorciada, un hijo).

(...) la renovación, para el reingreso vigente (del SNI), pues sí genera cierto, cierto estrés, ¿no? Incluso también con el proyecto que me han autorizado. Luego pone una sus metas, y que un libro, un artículo y todo eso y se te pasa el tiempo y que el libro no sale de la editorial y que tengo que presentar las evidencias, entonces andas también así un poquito agobiada con esas cuestiones (...). Yo tuve bruxismo de tanto que apretaba la mandíbula (...). Por las cuestiones de estrés a las que te enfrentas (...) (Elisabeth, casada, dos hijas).

Las académicas han reconocido el riesgo y las consecuencias que representa su ritmo de vida, pero ello no les ha ayudado a escapar de las presiones y el sedentarismo. El estilo de vida insano se acentúa por la falta de tiempo, las demandas y las formas en las que se requiere llevar a cabo el trabajo.

(...) he subido mucho de peso (...) son varias horas sentada y todo eso pero a veces dices, bueno, me voy al gimnasio, me voy a correr, voy a hacer esto y de aquí a que otra vez vuelves a agarrar el hilo de lo que estabas trabajando y escribiendo, a veces dices, "no, mejor aquí me quedo y termino, y a veces estás así como que muy sedentaria, ¿verdad? No me he checado últimamente (...) pero sí no me siento, no me siento saludable, ¿verdad? Obviamente, sé que si sigue una con ese ritmo de vida, bajo ciertas presiones y muy sedentario, pues obviamente tarde o temprano se va a ver reflejado, ¿no? y los análisis van a decir otra cosa (Elisabeth, casada, dos hijas).

Asumir con mayor responsabilidad ciertas prácticas de autocuidado ha sido una preocupación de parte de algunas de las académicas. Con el paso del tiempo, han reconocido la importancia del descanso no condicionado, tener una buena alimentación, gozar de un estado emocional equilibrado y, en general, llevar una supervisión y un control preventivo de su salud.

(...) va aprendiendo uno (sic) en el transcurso de los años, yo creo, y más en cuanto va uno (sic) creciendo o envejeciendo, más, ¿verdad?, 
que tienes que irte consintiendo y cuidando tú sola, ¿no? y más estando sola (...) (María, soltera, sin hijos)

(...) ahora sí me puedo cuidar más, si me quiero dormir un rato a medio día, me puedo acostar, o si necesito descansar un tiempo porque me siento cansada, pues puedo hacerlo. (...) no tengo la presión de la familia y eso pues se me hace increíble (Cristina, viuda, tres hijos, tres nietos).

(...) afortunadamente mi salud hasta ahorita está bien. Trato siempre, generalmente, de dedicar tiempo para hacerme mis controles preventivos (...). Afortunadamente, te digo, hasta ahorita no tengo nada así, problemas serios de salud, un problema crónico, no. La alimentación trato también de más o menos llevarla bien y en mi aspecto emocional como que me considero una persona equilibrada (...) Yo siento que conforme va pasando (el tiempo), la edad me ha vuelto así (...) (Margarita, soltera, una hija).

Los tres relatos anteriores pertenecen a mujeres solteras o viudas. La coincidencia introduce cuestionamientos sobre la injerencia del rol de pareja en las conductas de autocuidado de las mujeres.

En estos relatos, las participantes no solo están conscientes del valor que adquiere su salud sino también dejan ver la importancia de contar con un programa de atención y prevención como práctica de control. Pese a ello, la mayoría de las participantes se han desenvuelto como mujeres multi rol y multi tareas, en condiciones no del todo favorables para su salud y bienestar.

Ante momentos de conflicto y tensión, agenciarse y construir soportes psicológicos les han permitido protegerse y mitigar situaciones adversas. Especialmente cuando reconocen su propio poder y se responsabilizan de su vida.

La construcción social en torno a que las parejas deben cuidad a la familia dada la asignación de los roles de la maternidad y la paternidad puede llevar a que tanto mujeres como hombres deleguen en su pareja el cuidado de sí. Ante ello, cabe preguntar si mujeres y hombres fantasean tener una pareja que se preocupe por ellas y no se dan cuenta que ésta está asume la misma idea. 


\section{Soportes psicosociales}

Algunas de las participantes han buscado apoyos psicológicos profesionales para sobrellevar las cargas emocionales de la vida cotidiana. Si bien no han solucionado por completo sus malestares, les han permitido sobrellevar los problemas, tal como lo han hecho otras mujeres trabajadoras que han implementado estrategias mediante la autoatención y el apoyo social (Berenzon-Gorn, Saavedra-Solano, \& Alanís-Navarro, 2009).

Así, trabajar lo personal a nivel emocional ha permitido disipar parte del estrés. El apoyo profesional ha sido uno de esos soportes en busca del equilibrio y de respuestas a ciertos conflictos y cuestionamientos en torno a sus roles.

si no hubiera yo tenido esa parte de trabajo personal y de trabajar en lo emocional, yo no creo que hubiera avanzado como lo he hecho hasta ahorita (...) tuve que recurrir a ese apoyo (psicoterapéutico) (...) ahí es donde tienes oportunidad de recuperar esta parte de qué es lo que quieres hacer y si así es como quieres seguir y si así es como quieres avanzar (...), si quiero seguir siendo mamá, si quiero seguir (...) con pareja o no. Porque ese es otro esfuerzo más (...) (Elvira, casada, dos hijos).

Ciertos mecanismos para afrontar los problemas pueden entenderse como ejercicios terapéuticos en la medida que posibilitan la auto-escucha y el auto-descubrimiento. La expresión de lo interior es así un ejercicio de auto-interlocución, auto-control o auto-liberación, por lo que, para tolerar los síntomas de los malestares resulta útil inventar nuevas prácticas, tal como aparece en el siguiente relato.

(...) tengo una ¿cómo se puede decir? Manía (...) después él (psicólogo) me la hizo ver (...). Cuando yo tengo broncas escribo, entonces me siento y escribo todas las broncas que tengo (...). Empecé con eso hace 20 años cuando mi mamá murió (...) aprendí a escribir cartas. Cuando tengo un problema laboral, un problema del que sea que me siento afectada, (me pongo) a escribir, y entonces saco ahí todas mis tensiones (Carlota, casada, dos hijo, un nieto). 
Restringir el tiempo de socialización ha resultado perjudicial, sobre todo cuando las horas del trabajo, las del cuidado de la familia y las del estudio impiden o dificultan mantener redes de amistad fuera del círculo de la familia y el ambiente laboral porque no hay permiso para salirse de las responsabilidades.

(...). El día nada más tiene 24 horas y entonces si se dedica la jornada matutina al trabajo y la vespertina a los hijos y la nocturna a estudiar, bueno, no es fácil encontrar días de 30 horas ¿no? para hacer otras actividades. Entonces yo creo que la restricción más importante es en la socialización fuera del círculo al que se pertenece (...). Entonces, en ese caso creo que eso sería lo (sic), en donde tiene mayor impacto (Clarissa, casada, tres hijos, dos nietos).

Cuando se ha contado con la oportunidad de llevar a cabo encuentros cercanos y sanadores de sí en el contexto laboral, la interacción con esa compañía ha resultado ser un apoyo sustantivo para la salud mental de las participantes.

(...) La nueva red trajo nuevos contactos, nuevas amigas $\mathrm{y}$ compañeras de proyecto (...) encontré una especie de hermanas mayores. En el transcurso de estos años nos hemos encontrado con algunas trabas y con situaciones tensas tanto en nuestra casa de estudio como en nuestra institución de adscripción. Creo que, su apoyo es vital para mantener la salud mental y ayudar a mantener la fuerza y la perspectiva (...) (Karen, casada, sin hijos).

La socialización ha resultado ser un recurso clave en la vida de estas académicas. Gozar de buena salud significa también la búsqueda de un desarrollo equilibrado de la vida en las relaciones interpersonales, familiares y de integración social (OMS, 2001). De esa forma, los apoyos sociolaborales han demostrado ser fuentes protectoras para la salud y el bienestar. Los soportes emocional y práctico permiten alcanzar un mayor control en el proceso de la toma de decisiones (Feldman, \& Saputi, 2007; Gómez, Pérez, Feldman, Bajes, \& Vivas, 2000). 


\section{Conclusiones}

El género es un determinante social de la salud que debe tomarse en cuenta para la evaluación de los indicadores particulares de la calidad de vida, tanto por las condiciones derivadas de los roles desempeñados, como por los elementos que operan en la construcción sociocultural identitaria de mujeres y hombres.

Las consecuencias de la doble presencia de las mujeres en los ámbitos del trabajo y la familia (Guzmán, et al., 2016), y su relación con la salud, resultan complejas. Por un lado, el trabajo remunerado refuerza el bienestar de las mujeres al aportarles un espacio para su desarrollo personalprofesional, autonomía económica, relaciones sociales, entre otros beneficios. Por otro, las responsabilidades aumentan el estrés, la fatiga y los hábitos no saludables. No obstante, la oportunidad de contar con recursos personales y laborales ofrece a las académicas la posibilidad de alcanzar mejores niveles de salud.

La academia aparece como un trabajo que estimula y estresa al mismo tiempo. Las profesoras-investigadoras-esposas-cuidadoras han tenido que desarrollar mecanismos psicológicos que les permitan hacer carrera a la vez que atienden sus múltiples roles. Las narrativas permitieron describir la despersonalización hacia sí mismas, la normalización del dolor, y las racionalizaciones que se expresan o al minimizar los síntomas y problemas de salud o al asumir que el estilo de vida que se lleva es saludable -aun cuando se tiene una vida sedentaria, estresante, con síntomas físicos y/o emocionales expresos-. Estos mecanismos parecen acentuarse en los periodos más estresantes y, juntos, se vinculan a la falta de autocuidado, a la negligencia hacia la salud propia, al incremento de la vulnerabilidad y del riesgo de desarrollar enfermedades.

Los relatos han dejado ver cómo el estrés multitareas se relaciona con las enfermedades y dolencias tales como el sedentarismo, la obesidad, los dolores musculoesqueléticos, la hipertensión, la fatiga crónica, los dolores de cabeza, el estrés reactivo, la diabetes, la ansiedad; pero también se ha atenuado gracias a algunos soportes psicosociales como los apoyos profesionales (psicoterapéuticos), la interacción social al interior y al exterior del entorno laboral, los apoyos domésticos y los institucionales, los procesos de auto examinación y auto interlocución. 
Reconocer las tensiones no siempre ha redundado en atacar los estresores primarios. Ser consciente de las inequidades de género, no siempre implica la consecución de la equidad. El grado educativo y el puesto académico no eliminan las presiones de ser mujer. Lo expuesto aquí sugiere que, como resultado de las presiones socioculturales, las académicas pueden anteponer los requerimientos laborales y la atención de las necesidades de otros a sus propias necesidades de salud.

El análisis de la situación de este grupo de académicas obliga a tomar en cuenta diversas condiciones: que mujeres y hombres son distintos biológicamente; que los papeles psicosociales son diferenciados hasta hoy; que las condiciones laborales no responden a las demandas de sus múltiples roles y a la serie de problemas cotidianos para hacer compatible la vida laboral y familiar; que las imágenes sociales suelen ser contradictorias, ya que ser buena madre o buena hija - asumir cargas, culpas y sacrificio resulta difícil de compatibilizar con la imagen de la mujer como persona y su derecho a definir por sí misma un proyecto de vida como profesional en la academia y la investigación.

La lente de género permite comprender las relaciones que se dan entre la salud y la sociedad, al tiempo que compromete en la promoción de políticas sanitarias más adecuadas que beneficien a las personas. La igualdad de género implica la idea de que todos los seres humanos, mujeres y hombres, son libres para desarrollar sus capacidades, atender sus necesidades personales y tomar sus decisiones. Para lograr esa igualdad se requiere de la equidad como un ejercicio de justicia en el tratamiento a mujeres y a hombres, de acuerdo con sus respectivas necesidades (UNICEF y OIT, 2013).

En la incorporación de las mujeres al espacio público se debe promover la adopción de hábitos y estilos de vida no dañinos, así como prácticas que les permitan delegar tareas doméstico-familiares para hacerse de un tiempo personal en su autocuidado y control de la salud; el reconocimiento de la gravedad de sus síntomas y enfermedades; las visitas regulares al médico, la disciplina de una buena alimentación y un adecuado programa de ejercicios, apoyo psicoterapéutico y fortalecimiento de sus redes de socialización personales.

El problema de salud en este grupo de académicas tiene que ver, desde una perspectiva contractual-laboral, con el papel que le corresponde 
asumir a la universidad. Por lo tanto ¿Qué debe hacer la institución con respecto a la salud y el autocuidado de su personal académico? ¿Qué les corresponde hacer a las académicas y qué a sus centros de trabajo? ¿Qué acciones se deben llevar a cabo para prevenir y controlar aspectos de trabajo que ponen en riesgo la salud de las académicas? ¿Cómo implementar un programa, con perspectiva de género, para la promoción de la salud y prevención de enfermedades en el personal académico? ¿De qué forma se puede realizar una permanente y adecuada evaluación de la salud de las académicas? ¿Cómo se puede definir un programa de educación en salud con medidas de higiene y seguridad en el trabajo con impacto positivo en la vida personal de las académicas? ¿Qué políticas de conciliación laboral son necesarias a favor de quienes colaboran en los cuidados familiares?

Las tareas y los tiempos de las mujeres trabajadoras, más que las de los hombres, están llenos de horarios rígidos y obligaciones difícilmente evitables que concilian mal con las demandas de las instituciones donde laboran. Hay malos arreglos femeninos entre el tiempo y el trabajo que las organizaciones no están tomando en cuenta (Torns, 2005). Se carece de políticas de conciliación enfocadas a la compatibilidad familia-trabajo que repercutan en el bienestar cotidiano de las mujeres, de aquellas personas que lo reciben y del entorno. Es evidente que, en las llamadas sociedades del bienestar, las condiciones de vida actuales hacen persistentes las desigualdades de género dada la división sexual del trabajo (Crompton y otras, 2005, en Torns, 2014).

Los planteamientos que se han expuesto hasta ahora no agotan la comprensión del problema circunscrito en torno a la salud y el autocuidado del grupo de académicas participantes; sin embargo, permiten reconocer el género como uno de los determinantes más significativos en la vivencia de las mujeres. El estudio de las desigualdades genéricas y la salud precisa de un enfoque integral no solo cualitativo sino también cuantitativo para explicar y comprender los hechos sociales, y el sentido que los sujetos le dan a sus acciones. Esfuerzo científico que seguramente abrirá más posibilidades para trascender los límites que este trabajo presenta en su abordaje cualitativo. 


\section{Referencias}

Alderete, M.G., \& Franco, S.A. (2006). Una aproximación al análisis del trabajo de las mujeres desde la perspectiva de género. En: Factores Psicosociales y salud mental en el trabajo (Primera edición). México: U de $\mathrm{G}$.

Arquero, J.L., \& Donoso, J.A. (2013). Docencia, investigación y burnout: el síndrome del quemado en profesores universitarios de Contabilidad. Spanish Accounting Review 16 (2), 94-105

Armenti, C. (2004).Women faculty seeking tenure and parenthood: Lessons from previous generations. Cambridge Journal of Education, 34(1), 6583.Doi: 10.1080/0305764042000183133

Barbera, N. \& Iniciarte, A. (2012). Fenomenología y hermenéutica: dos perspectivas para estudiar las ciencias sociales y humanas. Multiciencias, 12(2), 199-205.

Barraza, H., \& Romero, J.J. (2011, febrero-marzo). Riesgos psicosociales y daños a la salud en el trabajo. Estudio de caso en el personal académico de la Facultad de Medicina de la Universidad Autónoma de Nayarit. Synthesis, 59, 34-39.

Bertaux. D. (1980/1990, marzo). El enfoque biográfico: su validez metodológica, sus potencialidades. Traducción hecha por el TCU 0113020 de la Universidad de Costa Rica de: "L'approche biographique: Sa validité méthodologique, ses potentialités", publicado originalmente en Cahiers Internationaux de Sociologie, 69,197-225. Revista Proposiciones, 1-23.

Berenzon-Gorn S, Saavedra-Solano N, \& Alanís-Navarro S. (2009, noviembre-diciembre). Estrategias utilizadas por un grupo de mujeres mexicanas para cuidar su salud emocional: autoatención y apoyo social. Salud Pública, 51, 474-481.

Blanco, G., \& Feldman, L. (2000, mayo/junio) Responsabilidades en el hogar y salud de la mujer trabajadora. Salud Pública de México, 42(3), 217-225.

Camacho Cristiá, C., \& Arias Galicia, F. Salud y agotamiento profesional (Burnout) en profesores veracruzanos. En: Agotamiento profesional y estrés, hallazgos desde México y otros países latinoamericanos. Arias 
Galicia, F., \& Juárez García, A. México: Universidad Autónoma del Estado de Morelos -Porrúa, 14-40.

Carrasquer, P. (2009). La doble presencia. El trabajo y el empleo femenino en las sociedades contemporáneas. (Tesis de doctorado por compilación de publicaciones). Departamento de Sociología, Universidad Autónoma de Barcelona. Barcelona, España.

Díaz, N. (1999). El relato de una vida: apuntes teóricos-metodológicos en comunicación. Revista Latina de Comunicación Social, 22, Recuperado de:

http://www.ull.es/publicaciones/latina/biblio/valencia99/33vanancy.html

Esteban Galarza. M. L. (2003). El género como categoría analítica. Revisiones y aplicaciones a la salud. Cuadernos de Psiquiatría Comunitaria, 3(1), 22-39.

Feldman, L., \& Saputi, D. (2007). Roles múltiples, cualidad del rol, apoyo social y salud en mujeres trabajadoras. Revista Venezolana de Estudios de la Mujer, 12(29), 91-119.

Gómez, V. (2004). Estrés y salud en mujeres que desempeñan múltiples roles. Avances en Psicología Latinoamericana, 22, 117-128.

Gómez, V., Pérez, L.A., Feldman, L., Bajes, N., \& Vivas, E. (2000, mayo). Riesgos de salud en mujeres con múltiples roles. Revista de Estudios Sociales, 9, 27-38.

Gómez, V., Perilla, L. \& Hermosa, A. M. (2015) Moderación de la relación entre tensión laboral y malestar de profesores universitarios: papel del conflicto y la facilitación entre el trabajo y la familia. Revista Colombiana de Psicología, 24(1), 185-201.

González-Pier E, Gutiérrez-Delgado C, Stevens G, Barraza-Lloréns M, Porras-Condey R, Carvalho N et al. (2006). Priority setting for health interventions in Mexico's System of Social Protection in Health. The Lancet, 368, 1608-1618.

Guzmán, B. M. de R., Polanco, G., Ulloa, C., \& Rocha, T.E. (2016). Conciliación doble-presente en mujeres académicas universitarias. (Artículo en proceso para su publicación).

Instituto Nacional de Geografía e Informática e Instituto Nacional de las Mujeres. (2014). Resultados de la Encuesta Nacional sobre uso del tiempo. Boletín de prensa No. 273/15, 13 de julio de 2015, Aguascalientes, Ags. Consultado el 10 de noviembre de 2016. 
1679 Gúzman, Polanco, Reynoso \& Maldonado - Género, Salud y Autocuidado

Recuperado en: http://consulta.mx/index.php/estudios-einvestigaciones/otros-estudios/item/723-inegi-e-inmujeres-encuestanacional-sobre-uso-del-tiempo-2014

Magaña, D.E., Sánchez, P.A. (2008). Síndrome de Desgaste Emocional en Investigadores Mexicanos. Interamerican Journal of Psychology, 42 (2) 2008, 353-362.

Maslach, C. (2003). Job Bournout: new directions in research and interventions. American Psychological Society, Current directions in psychological science, 12 (5).

Moreno, B., González, J.L., \& Garrosa, E. Desgaste profesional en hospitales: influencia de las variables socio demográficas. Rol de Enfermería, 25, 19-26.

Organización Mundial de la Salud. (2001, marzo). La salud mental en las Américas: Nuevos retos al comienzo del milenio. Documento presentado a la Sesión del Subcomité Ejecutivo de Planificación y Programación de la Oficina Panamericana de la Salud. Washington: OPS.

Organización Mundial de la Salud. (2002). Integración de las perspectivas de género en la labor de la OMS. Política de la OMS en materia de género. Londres: OMS.

Organización Mundial de la Salud. (2004). Invertir en salud mental. Suiza: OMS.

Organización Mundial de la Salud. (2011). Informe sobre el Sistema de salud Mental en México. México: OMS, OPS, Secretaria de Salud.

Palomino, P., Grande, M.L., \& Linares, M. (2014, junio). La salud y sus determinantes sociales. Desigualdades y exclusión en la sociedad del siglo XXI. Revista Internacional de Sociología (RIS), 2(1), 71-91. DOI:10.3989/ris.2013.02.16

Probert, B. (2005). "I just couldn't fit in": Gender and unequal outcomes in academic careers. Gender, Work, and Organization, 12(1), 50-72.

Reddick, R., Rochlen, A., Grasso, J., Reilly, E., \& Spikes, D. (2012). Academic fathers pursuing tenure: A qualitative study of work-family conflict, coping strategies, and departmental culture. Psychology of Men \& Masculinity, 13(1), 1-15. DOI: 10.1037/a0023206

Scott, J. (2013). El género: Una categoría útil para el análisis histórico. En: M. Lamas (comp.), El género. La construcción cultural de la diferencia sexual (pp. 265-302). México: Porrúa, PUEG-UNAM. 
Tena, O., Muñoz, A.L., \& López, J. (2012, marzo-agosto). Productividad académica, maternidad y malestares subjetivos en investigadoras e investigadores de la Universidad Nacional Autónoma de México. GénEros, 11(2), 57-78.

Tena, O., Rodríguez, C., \& Jiménez, P. (2010, marzo). Malestares y uso del tiempo en investigadoras de la Facultad de Estudios Supriores (FES) Iztacala. Investigación y Ciencia de la Universidad Autónoma de Aguascalientes, 46, 65-75.

Taylor, S.E., Kemeny, M.E., Reed, G.M., Bowel, J. E., \& Gruenewald, T.L. (2000). Psychological resourses positive illusions, and health. American Psychologist, 55(1), 99-109.

Torns, T. (2011, diciembre). Conciliación de la vida laboral y familiar o corresponsabilidad: ¿El mismo discurso? Revista Interdisciplinaria de Estudios de Género, 1, 5-13.

Wiersma, U., \&Van Den Berg, P. (1991). Work-home role conflict, family climate, and domestic responsabilities, among men and woman in dualearner families. Appl. Soc. Psychol, 21(15), 1207-1217.

Vázquez, C., Hervás, G, Rahona, J.J., \& Gómez, D. (2009). Bienestar psicológico y salud: Aportaciones desde la psicología positiva. Anuario de Psicologia Clínica y de la Salud, 5, 15-28.

María del Rocío Guzmán Benavente Profesora-Investigadora de la Facultad de Psicología y Terapia de la Comunicación Humana. Universidad Juárez del Estado de Durango, México.

E-mail address: marociogb@ujed.mx 